\title{
THE CHOICE BETWEEN COOLED TUBULAR REACTOR MODELS: ANALYSIS OF THE HOT SPOT
}

\author{
E. J. WESTERINK, N. KOSTER and K. R. WESTERTERP ${ }^{\dagger}$ \\ Chemical Reaction Engineering Laboratories, Department of Chemical Engineering, Twente University of \\ Technology, PO Box 217, 7500 AE Enschede, Netherlands
}

(Received 31 May 1988; accepted for publication 24 April 1990)

\begin{abstract}
The applicability of the one-dimensional pseudo-homogeneous model of the cooled tubular reactor is studied. Using the two-dimensional model as the more accurate one we compared both models by studying the influence of the design and operating variables on the conditions in the hot spot of the reactor. The effects were studied on an analytical basis, and a relation is derived that describes the radial temperature profile in the hot spot of the reactor. In the first section we present the model equations and discuss the results obtained from a numerical evaluation. In the second section we compare mean and maximum radial temperatures and reaction rates in case a single exothermic reaction is carried out. We conclude that - for reactors operating in the steady state - in the hot spot the one-dimensional model predicts the proper temperature when it is compared with the average temperature calculated by the twodimensional model, although large differences may arise between maximum and mean radial temperature. A new method is presented to obtain the maximum radial temperature in the hot spot directly from the results of the one-dimensional model. It was found that there can be large differences between the actual average reaction rate and the reaction rate at mean temperature as obtained from the one-dimensional model.
\end{abstract}

\section{INTRODUCTION}

In the literature many studies have been published dealing with the proper design of tubular reactors for exothermic reactions. These studies are either based on the prevention of runaway or the preservation of a certain a priori desired selectivity. Well known is the paper by Barkelew (1959), who showed that in a plot of the conversion $v s$ the hot spot temperature two regions can be distinguished. One region-that of the low parametric sensitivity-has the property that a small change in the operating conditions also leads to a small change in the hot spot temperature. In the second region - that of high parametric sensitivity - a small change in operating conditions causes a large increase in the temperature profile along the reactor axis.

Westerterp et al. (1984), Westerterp and Overtoom (1985), and Westerink and Westerterp (1988) present a series of papers dealing with the design and operation of cooled tubular reactors for reaction systems consisting of two or more exothermic reactions and relate the design and operating parameters to the desired selectivity. Both Barkelew and Westerterp used the pseudo-homogeneous one-dimensional model (ODM) to arrive at their results.

Morbidelli and Varma $(1988,1989)$ presented a series of papers in which they study parametric sensitivity in a cooled tubular reactor. They conclude that the reactor becomes sensitive to all the operating conditions and physicochemical parameters simultaneously.

\footnotetext{
${ }^{\dagger}$ Author to whom correspondence should be addressed.
}

Several investigators presented papers that deal with the applicability of the ODM. Froment (1967, 1974) studied the difference between the ODM and the pseudo-homogeneous two-dimensional model (TDM). He concluded that for "mild conditions" the ODM predicts the proper mean temperature. Froment does not point out what "mild conditions" are but suggests using the TDM in view of the increased power of the computer.

Hlavacek (1970) also showed that the ODM gives proper values of the mean radial temperature as long as the reactor is operated in the region of low parametric sensitivity of the TDM. He points out that the regions of parametric sensitivity of the TDM and ODM differ. Both Hlavacek and Froment based their results on a semi-theoretical basis: they developed parameters and scanned the region using the numerically solved TDM.

Mears (1971) presented a criterion based on an analytical solution of the radial profile in a packed bed as derived by Chambré and Grossman (1955). He combined the mean reaction rate, the wall temperature, the effective conductivity in the bed and the activation temperature into a relation to be fulfilled for a quasi-isothermal radial temperature profile.

In this study we will focus mainly on the temperature and reaction rate profiles in the hot spot of the reactor, where the conditions are extreme, and compare the behavior of the ODM and the TDM under equal conditions. First, we will discuss the results obtained from the model equations of the TDM and the ODM. Next, we will derive a new radial temperature profile equation for the hot spot.

In the next section we will define regions for which the ODM is in good agreement with the TDM. Moreover, we will relate the radially mean temperature in 
the hot spot, as obtained from the ODM, to the maximum radial temperature. Apart from the temperature reaction rates are also important since they influence the selectivity. Therefore, we will also compare the mean reaction rate obtained from the TDM with the reaction rate calculated by the ODM.

\section{THE REACTOR MODELS USED FOR COMPARISON}

\section{The two-dimensional model}

For more complex reactor design problems the TDM is frequently used. For a single first-order exothermic reaction the following partial differential equations are obtained for the case when axial dispersion is neglected for the conversion:

$$
\begin{aligned}
\frac{\partial X_{A}}{\partial z}= & 4 L R D R B o_{m}^{-1} \frac{1}{r^{*}} \frac{\partial}{\partial r^{*}}\left(r^{*} \frac{\partial X_{A}}{\partial r^{*}}\right) \\
& +D a_{1}\left(1-X_{A}\right) \exp \left(\frac{\gamma_{P} \theta}{1+\theta}\right)
\end{aligned}
$$

and for the temperature:

$$
\begin{aligned}
\frac{\partial \theta}{\partial z}= & 4 L R D R B o_{h}^{-1} \frac{1}{r^{*}} \frac{\partial}{\partial r^{*}}\left(r^{*} \frac{\partial \theta}{\partial r^{*}}\right) \\
& -D a_{1}\left(1-X_{A}\right) \Delta \theta_{\mathrm{ad}} \exp \left(\frac{\gamma_{P} \theta}{1+\theta}\right)
\end{aligned}
$$

These equations are subject to the following boundary conditions:

$$
\left.\frac{\partial X_{A}}{\partial r^{*}}\right|_{r^{*}=1}=\left.0 \quad \frac{\partial \theta}{\partial r^{*}}\right|_{r^{*}=1}=B i \theta(\vec{r}=1) \forall z
$$

and

$$
\left.\frac{\partial X_{A}}{\partial r^{*}}\right|_{r^{*}=1}=\left.0 \quad \frac{\partial \theta}{\partial r^{*}}\right|_{r^{*}=0}=0 \quad \forall z .
$$

The initial conditions are

$$
\begin{aligned}
& \theta=\theta_{o}=\theta_{c}=0 \quad \text { for } z=0 \quad \forall r^{*} \\
& X_{A}=0 \quad \text { for } z=0 \quad \forall r^{*} \text {. }
\end{aligned}
$$

The most important parameters are the tube slenderness $(L R)$, the reciprocal number of particles on a diameter $(D R)$, the Bodenstein numbers for mass and heat $\left(\mathrm{Bo}_{m}\right.$ and $\left.\mathrm{Bo}_{h}\right)$, the Biot number $(\mathrm{Bi})$, the adiabatic temperature rise $\left(\Delta \theta_{\mathrm{ad}}\right)$ and the dimensionless residence time $\left(D a_{1}\right)$. Refer to the notation for their definitions.

The values of $B o_{m}, B o_{h}$ and $B i$ have to be determined from empirical correlations. We performed many profile computations and scanned the following parameter ranges:

$$
\begin{aligned}
10 & <B o_{m}<20 \\
8 & <B o_{h}<15 \\
1 & <B i<10 \\
0.5 & <L R D R<20 .
\end{aligned}
$$

Moreover we choose $D a_{1}=100$ to achieve nearly complete conversion and $\Delta \theta_{\text {ad }}=1.5$ to obtain rather pronounced temperature profiles. We have used the relations presented by Fahien and Smith (1955) for the dispersion of mass, and by Hennecke and Schlünder (1973) and Zehner and Schlünder (1973) for the dispersion of heat. For the calculation of $\lambda_{\text {eff }}$ we refer to the work of Hennecke, Zehner and Schlünder (1973) [also see Westerterp et al. (1984)]. Bi number was calculated from the Dixon and Cresswell (1979) correlation.

Usually the dispersion factor for mass, $B o_{m}$, is 10 . According to the relation of Fahien and Smith (1955) it is corrected for the number of particles on a diameter. If $D R$ is high $B o_{m}$ increases, so we assumed a maximum value of $D R=1 / 4$ which leads to a maximum value of $B o_{m}=20$. The dispersion factor for heat, $B o_{h}$, is affected by both the thermal conductivity of the stagnant bed, $\lambda_{o}$, and the heat dispersion due to flow, $\lambda_{l}$. For the conductivity due to flow $B o_{h} \approx 8$, and also this number depends on the number of particles on a diameter and it increases for increasing $D R$. For the maximum value of $D R$ we obtain a value $B o_{k}=15$.

We found the following effects after evaluation of the results:

(1) For all parameter values given above the radial concentration gradient can be neglected. Among others this was also found by Froment (1974). This can be understood if we take into account that mass can not pass through the reactor tube wall, so there is no driving force for mass towards the wall. This is in contrast to heat, which does pass the reactor tube wall.

(2) For parameter values up to conditions that lead to extreme hot spot temperatures-so near runaway conditions - the radial location of the hot spots all are in the same plane perpendicular to the tube axis.

\section{The one-dimensional model}

The ODM is the most popular model for the design of cooled tubular reactors since it is easy to solve and manipulate in a mathematical sense. The model equations can be obtained from the TDM by substituting radially averaged values for the temperature and concentration. The following equations are obtained

for the conversion:

$$
\frac{\mathrm{d} X_{A}}{\mathrm{~d} z}=D a_{1}\left(1-\left\langle X_{A}\right\rangle\right) \exp \left(\frac{\gamma_{P}\langle\theta\rangle}{1+\langle\theta\rangle}\right)
$$

and for the temperature:

$$
\begin{aligned}
\frac{\mathrm{d}\langle\theta\rangle}{\mathrm{d} z} & =D a_{\mathrm{I}} \theta_{\mathrm{ad}}\left(1-\left\langle X_{A}\right\rangle\right) \exp \left(\frac{\gamma_{P}\langle\theta\rangle}{1+\langle\theta\rangle}\right) \\
& -D a_{\mathrm{I}} U^{*}\langle\theta\rangle
\end{aligned}
$$

with usually the initial conditions $\theta=\theta_{o}=\theta_{c}=0$ and $X_{A}=0$ for $z=0$.

In the TDM the withdrawal of heat is described by a conductivity in the bed $\left(\lambda_{\text {eff }}\right)$ and a transfer of heat 
at the reactor wall $\left(\alpha_{w}\right)$. In the ODM these effects are lumped into one parameter called the overall heat transfer coefficient $U$. There are several relations given for the lumping of $\alpha_{w}$ and $\lambda_{\text {eff }}$. Crider and Foss (1965) suggested that

$$
\frac{U}{\alpha_{w}}=\frac{1}{1+B i / 3.06}
$$

whereas Beek (1962) proposed

$$
\frac{U}{\alpha_{w}}=\frac{1}{1+B i / 4.0} .
$$

Both relations differ only in the factor 3.06 or 4.0 in the denominator on the right-hand side. Of course the conversion and temperature profiles calculated by the ODM will depend on which relation is used.

The model equations of the ODM can not be solved analytically. They can be reduced to only one by dividing eq. (4) by eq. (3) which leads to the equation describing the so-called trajectories:

$$
\begin{gathered}
\mathrm{d}\langle\theta\rangle \\
\mathrm{d}\left(1-X_{A}\right)
\end{gathered}=\Delta \theta_{\mathrm{ad}}-\frac{\langle\theta\rangle U^{*}}{1-X_{A}} \exp \left(\begin{array}{c}
-\gamma_{P}\langle\theta\rangle \\
-1+\langle\theta\rangle
\end{array}\right) .
$$

For most chemical reactions and coolant temperatures the value of $\gamma_{P}$ varies from 10 up to 100 ; in these cases it is allowed to substitute $1+\theta$ by 1. This is the so-called Frank-Kamenetski approximation. It is convenient to combine the parameters $\gamma_{P}$ and $\langle\theta\rangle$ into one $\langle\varphi\rangle$. These substitutions lead to

$$
\frac{\mathrm{d}\langle\varphi\rangle}{\mathrm{d} z}=\gamma_{P} \Delta \theta_{\mathrm{ad}}-\frac{\langle\varphi\rangle U^{*}}{1-X_{A}} \exp (-\langle\varphi\rangle) .
$$

The Frank-Kamenetski approximation appears to be valid for cases where $\langle\varphi\rangle \leqslant 1$. To study the effects in the hot spot the conversion can be related to the hot spot temperature by putting $\frac{d\langle\varphi\rangle}{d z}=0$ in eq. (8), which gives us

$$
\left(1-\left\langle X_{A}\right\rangle\right)_{h s}=\frac{\langle\varphi\rangle_{h s} U^{*}}{\gamma_{P} \Delta \theta_{\text {ad }}} \exp \left(-\langle\varphi\rangle_{h s}\right) .
$$

\section{$A$ new radial model for the hot spot: the $R T P M$}

In our discussion above we derived the model equations for the ODM from the TDM mainly by substituting radially averaged values of the temperature and conversion. We will now derive a new model for the Radial Temperature Profile (RTPM) in the hot spot.

If we reexamine the conclusions of the numerical evaluations of the TDM the following simplifications can be made for the model equations of the TDM in the hot spot. We observed that for every radial position the hot spot was located at the same axial position, therefore-in the hot spot-the partial derivative $\frac{\partial \theta}{\partial z}$ in eq. (2) can be put equal to zero. Moreover we observed that there is no significant radial concentration gradient which makes it possible to introduce a radially mean conversion $\left\langle X_{A}\right\rangle$ as was done for the ODM. If also the Frank-Kamenetski approximation is used we obtain the following differential equation for the radial temperature profile in the hot spot:

$$
\begin{aligned}
& \frac{1}{r^{*}} \frac{\mathrm{d}}{\mathrm{d} r^{*}}\left(r^{*} \frac{\mathrm{d} \varphi}{\mathrm{d} r^{*}}\right) \\
&=\gamma_{P} \frac{D a_{1} \Delta \theta_{\mathrm{ad}}\left(1-\left\langle X_{A}\right\rangle\right)_{h s}}{4 L R D R} B o_{h} \exp (\varphi)
\end{aligned}
$$

which is subject to the boundary conditions

$$
-\left.\frac{\mathrm{d} \varphi}{\mathrm{d} r^{*}}\right|_{r^{*}=1}=B i \varphi\left(r^{*}=1\right) \text { and }\left.\frac{\mathrm{d} \varphi}{\mathrm{d} r^{*}}\right|_{r^{*}=0}=0 \text {. }
$$

If we rewrite eq. (10) using the definition of the fourth Damköhler group $D a_{\text {IV }}$ we get

$$
\frac{1}{r^{*}} \frac{\mathrm{d}}{\mathrm{d} r^{*}}\left(r^{*} \frac{\mathrm{d} \varphi}{\mathrm{d} r^{*}}\right)=\gamma_{P} D a_{\mathrm{rv}}\left(1-\left\langle X_{A}\right\rangle\right)_{h s} \exp (\varphi)
$$

From this equation we can see that the temperature profile is governed by the hot-spot conversion $\left\langle X_{A}\right\rangle_{h s}$, the ratio of heat liberation over the conductive transport of heat ( $\left.D a_{I v}\right)$, the Arrhenius number $\gamma_{P}$ and $B i$. This equation can be solved analytically, as shown in Appendix 1, leading to the following relation:

$$
\varphi=-2 \ln \left[A+\frac{\gamma_{P} D a_{\mathrm{TV}}\left(1-\left\langle X_{A}\right\rangle\right)_{h s}}{8 A}\left(r^{*}\right)^{2}\right]
$$

The integration constant $A$ is obtained from the implicit relation:

$$
\begin{aligned}
& \frac{2}{8 A^{2}} \\
& \frac{\gamma_{P} D a_{\mathrm{IV}}\left(1-\left\langle X_{A}\right\rangle\right)_{h x}}{2}+1 \\
& +B i \ln \left[A+\frac{\gamma_{P} D a_{\mathrm{IV}}\left(1-\left\langle X_{A}\right\rangle\right)_{h s}}{8 A}\right]=0 .
\end{aligned}
$$

To check whether the analytical solution is correct we calculated radial temperature profiles in the hot spot using the TDM for a chosen set of parameter values and next we calculated radial temperature profiles using the RTPM, into which a value of $D a_{\mathrm{g}}$ was substituted with the conversion $\left\langle X_{A}\right\rangle_{h s}$ as obtained by the TDM. It appeared that for the calculated values of $\left\langle X_{A}\right\rangle_{h s}$ two solutions for the integration constant $A$ are possible. These two solutions and the radial profile as calculated by the TDM are shown in Fig. 1. The solution given by the TDM is in the region of low parametric sensitivity. Figure 1 shows that only one of the two solutions gives the correct profile. It must be understood that-although our RTPM method as an approximation gives two possible solutions-the TDM can give one solution only because of all the initial and boundary values being fixed. In the conversion $\left\langle X_{A}\right\rangle_{\text {hs }}$, the Damköhler number $D a_{1 V}$ and the Arrhenius number $\gamma_{P}$ we combined several reaction and reactor properties such as temperature 


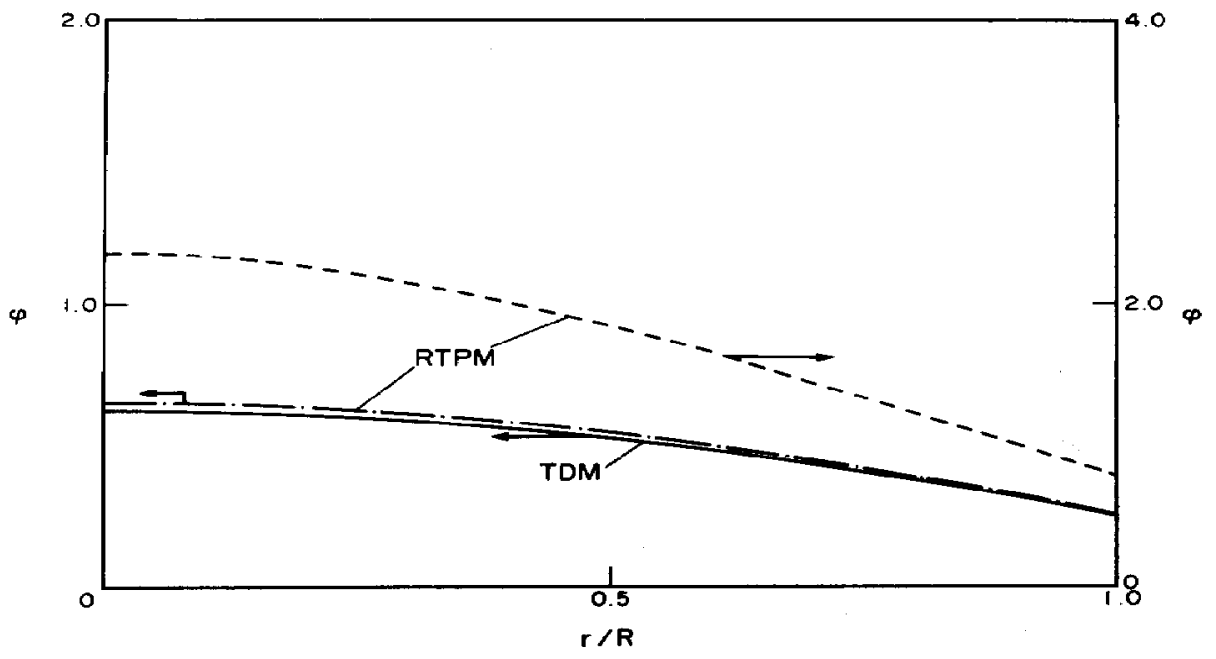

Fig. 1. Two temperature profiles given by the RPTM and the same in the hot spot as given by the TDM for the same conditions. The parameter $\gamma_{P} D a_{\mathrm{IV}}\left(1-\left\langle X_{A}\right\rangle\right)_{h s}$ is calculated using the mean conversion given by the TDM resulting in $\gamma_{P} D a_{\mathrm{rV}}\left(1-\left\langle X_{A}\right\rangle\right)_{k s}=0.9$. The values for $A$ are $A=0.9644$ and $A=0.0574$. For the TDM the parameters are: $\gamma_{F}=12.9, B i=2.77, B o_{m}=11.2, B o_{h}=9.6, \Delta \theta_{a d}=0.96, D R=0.08, L R=240$, $T_{c}=550, D a=1.02$.

sensitivity, thermal conduction and the reactant concentration. Each of these properties influence the profile in its own, but different, way. In the RTPM method where we use a certain reactant concentration $C_{A o}\left(1-\left\langle X_{A}\right\rangle\right)_{h s}$ in the hot spot that can be the result of a high inlet concentration and a low conversion in case of a well-cooled reactor or-also for the same $\gamma_{P} D a_{\Gamma V}\left(1-\left\langle X_{A}\right\rangle\right)_{h s}$ value-a low reactant inlet concentration and a high conversion which is the situation of near runaway in a badly cooled reactor. In studies of cooled tubular reactors-performed at our research institute-the factor $\gamma_{P} D a_{I V}\left(1-\left\langle X_{A}\right\rangle\right)$ is encountered frequently; it is the ratio of the temperature sensitivity of the heat production rate to that of the heat withdrawal rate.

In the case we are interested in mean temperatures these can be calculated by means of the following equation:

$$
\begin{aligned}
\langle\varphi\rangle_{h s} & =-2\left[1+\frac{8 A^{2}}{\gamma_{P} D a_{\mathrm{IV}}\left(1-\left\langle X_{A}\right\rangle\right)_{h s}}\right] \\
& \times \ln \left[1+\frac{\gamma_{P} D a_{\mathrm{IV}}\left(1-\left\langle X_{A}\right\rangle\right)_{h s}}{8 A^{2}}\right]+2-2 \ln (A)
\end{aligned}
$$

which is the solution of $\langle\varphi\rangle=\int_{0}^{1} \varphi r^{*} \mathrm{~d} r^{*} / \int_{0}^{1} r^{*} \mathrm{~d} r^{*}$

\section{ANALYSIS OF THE HOT SPOT}

\section{Locus of maxima curves}

In the previous paragraphs we described the three models that will be used for comparison. We will compare them by analyzing the conditions in the hot spot, which means the predicted hot spot temperatures for a given hot spot conversion. As a basis for our studies we use a plot of the hot spot conversion given by the variable $\gamma_{P} D a_{1 v}\left(1-\left\langle X_{A}\right\rangle\right)_{h s}$ vs the predicted mean hot-spot temperature $\langle\varphi\rangle_{h s}$. This method of representation is similar to that introduced by Barkelew (1959), who plotted $\varphi$ vs $X_{A}$.

In Fig. 2 we plotted two temperature profiles [(a) and (b)] calculated by the ODM using eq. (7) and eq. (5) for the lumping of $\lambda_{\text {efr }}$ and $\alpha_{w}$. Here $\gamma_{p} D a_{\mathrm{rv}}(1$ $\left.-\left\langle X_{A}\right\rangle\right)$ is used as a variable. The following relation exists between $D a_{1 v}$ and the model parameters of the ODM:

$$
D a_{\mathrm{IV}}=2 \frac{\Delta \theta_{\mathrm{ad}} B i}{U^{*}}\left(\frac{U}{\alpha_{w}}\right)
$$

As can be seen from Fig. 2, the profile starts at $\langle\varphi\rangle$ $=0$, going from right to left, it rises until the hot spot is reached and then decreases approaching the point reached after an infinite residence time and given by $\gamma_{P} D a_{I V}\left(1-\left\langle X_{A}\right\rangle\right)=0,\langle\varphi\rangle=0$. For the two profiles plotted the parameters were chosen such that profile (a) is in the region of low parametric sensitivity and profile (b) in the region of high parametric sensitivity. The dashed lines are the so-called locus of maxima curves, relating the temperature and the value of $\gamma_{P} D a_{\mathrm{IV}}\left(1-X_{A}\right)$ in the hot spot. Of course the locus of maxima curves can be calculated by means of any of the three reactor models mentioned. For the TDM and the RTPM we calculated the mean temperature in the hot spot using the FrankKamenetski approximation. We plotted the results of all three models for a given value of $\gamma_{P}$ in Fig. 3: it can be seen that the RTPM follows the curve of the TDM even beyond the maximum. By numerical evaluation it was found that in cases where the RTPM deviates from the TDM the radial concentration gradient is no longer flat; in that case the assumption of a constant 


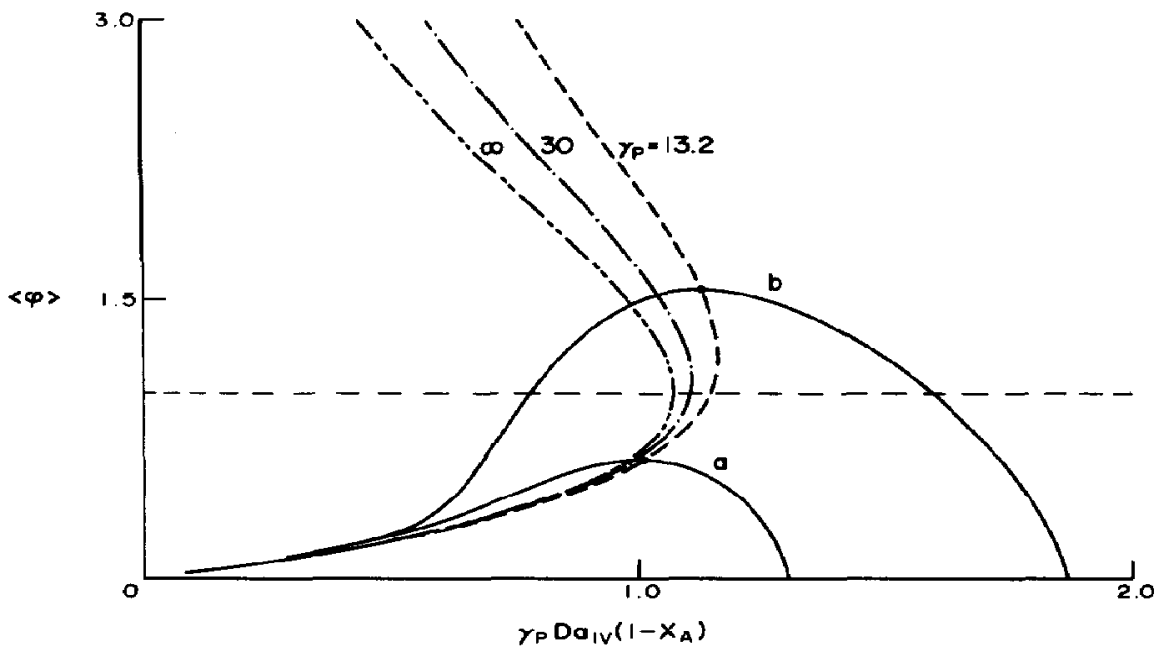

Fig. 2. Temperature $\langle\varphi\rangle$ as a function of the parameter $\gamma_{P} D a_{1 V}\left(1-\left\langle X_{A}\right\rangle\right)$. Two trajectories of the ODM

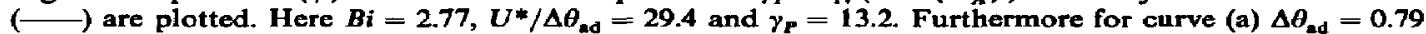
and $U^{*}=23.20$ whereas for curve (b) $\Delta \theta_{\text {ad }}=1.14$ and $U^{*}=33.50$. Curve (b) is already parametrically sensitive. Also the locus of maxima curves are given for the $O D M$ and $\gamma_{P}=13.2,30$ and $\infty$. In case the

Frank-Kamenetski approximation is used the locus curve with $\gamma_{F}=\infty$ is found for the $O D M$.

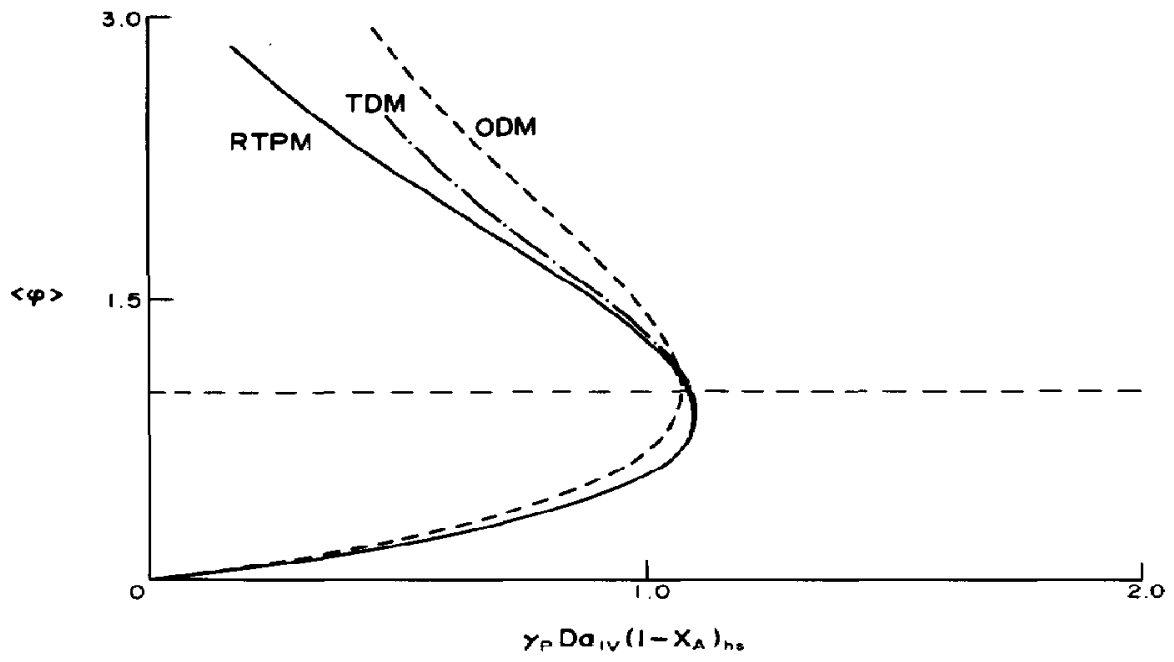

Fig. 3. Locus of maxima curves obtained with the TDM (- - - - -), the ODM (- - - - ) and the RTPM $(-)$ in case the Frank-Kamenetski approximation is used. Here $B i=2.77$.

concentration along the reactor radius is not valid anymore. However, the discrepancy occurs beyond the maximum in the locus of maxima curve which is beyond the point of practical interest because we then already operate in the undesired region of high parametric sensitivity. From this evaluation we may conclude that the RTPM and the TDM produce the same results as long as we operate in the region of low parametric sensitivity or, the other way around, the RTPM predicts the same region of low parametric sensitivity as does the TDM. This implies that the RTPM and the TDM are equivalent models as long as we can use the Frank-Kamenetski approximation
$\left(\gamma_{P}>10\right)$. Therefore in our discussion from now on we will use the RTPM instead of the TDM.

Differences in mean temperature as predicted by the $O D M$ and the RTPM

In Fig. 3 we plotted the locus of maxima curves of the three models for one explicit value of $B i, B i=2.77$. However, we wish to investigate the complete region of practical values of $B i, 1 \leqslant B i \leqslant 10$. Therefore we calculated the locus of maxima curves given by the RTPM and the ODM for $B i=1$ and $B i=10$. The results are shown in Fig. 4, where we used the lumping relation given by eq. (6). As can be concluded from 
Fig. 4 there are considerable differences in the predicted regions of low parametric sensitivity and the hot spot temperatures at a given conversion $X_{A}$. To illustrate the difference in the region of parametric sensitivity we plotted the maximum value of the parameter $\gamma_{P} D a_{\mathrm{IV}}\left(1-X_{A}\right)_{h s}$ as a function of $B i$. This is shown in Fig. 5, where the region of parametric sensitivity is below the plotted line-here the locus of maxima has two solutions, one in the region of high parametric sensitivity and one in the region of low parametric sensitivity. Above the line no solutions are possible, whereas only one solution is found for parameter values on the line. As can be seen in Figs 4 and 5 the predicted areas defined by the RTPM are much smaller then the ones predicted by the ODM. This implies that for design cases based on the ODM one might think that the parameter values found lead to safe operation while in reality they cause a runaway.

We also plotted the locus of maxima curves when eq. (5) is used for lumping of the heat parameters: this is shown in Fig. 6. As can be seen the differences between both areas prove to be much smaller than when eq. (6) was used for lumping. This is illustrated once more in Fig. 7. As can be concluded from Fig. 7 using eq. (5) for lumping leads to a good overall prediction of the area of safe operation. At low

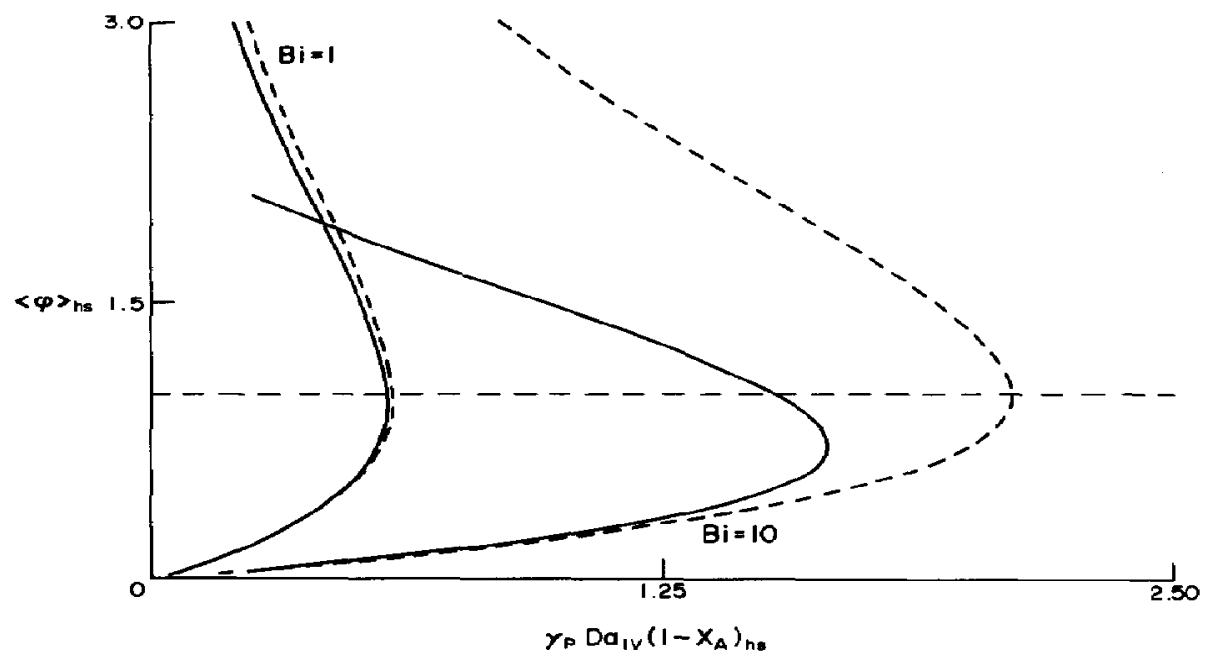

Fig. 4. Loci of maxima curves of the ODM (- - - ), for which the Frank-Kamenetski approximation is used, and the loci curves as found with the RTPM (__ ) for two values of Bi. Here 4.0 is used as a lumping factor.

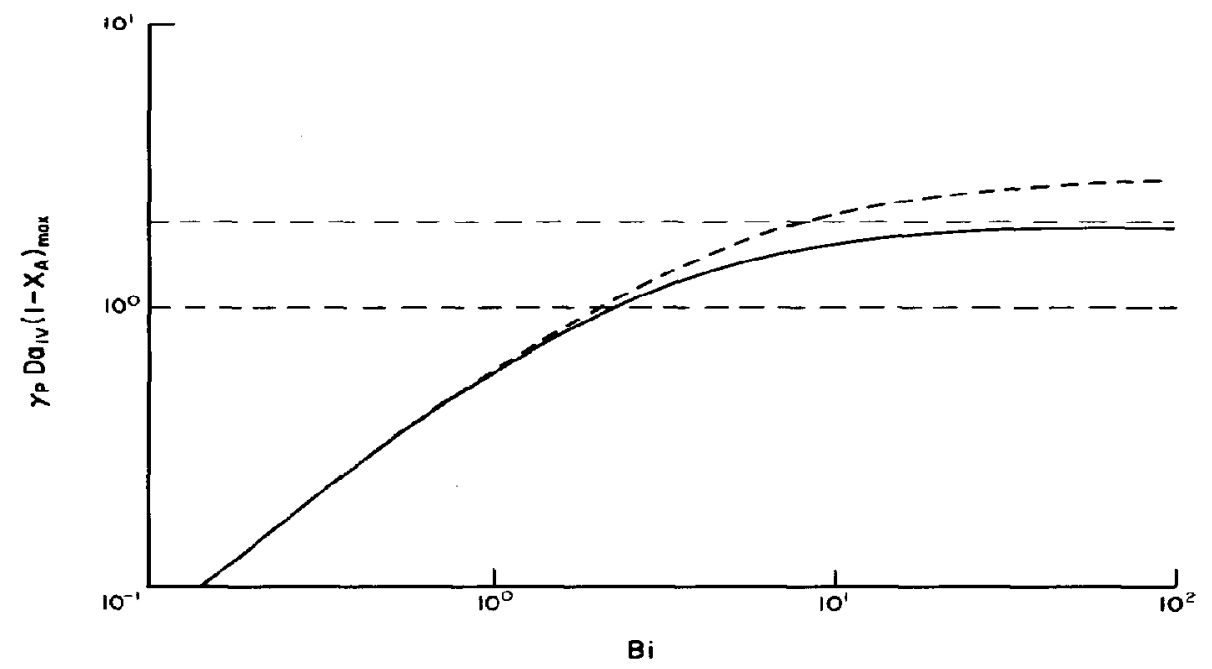

Fig. 5. $\left[\gamma_{P} D a_{1 \mathrm{v}}\left(1-\left\langle X_{A}\right\rangle\right)_{h s}\right]_{\max }$ as a function of $B i$ for the ODM $(---)$, with the Frank-Kamenetski approximation, and the RTPM (-). The lumping factor is 4.0 . 


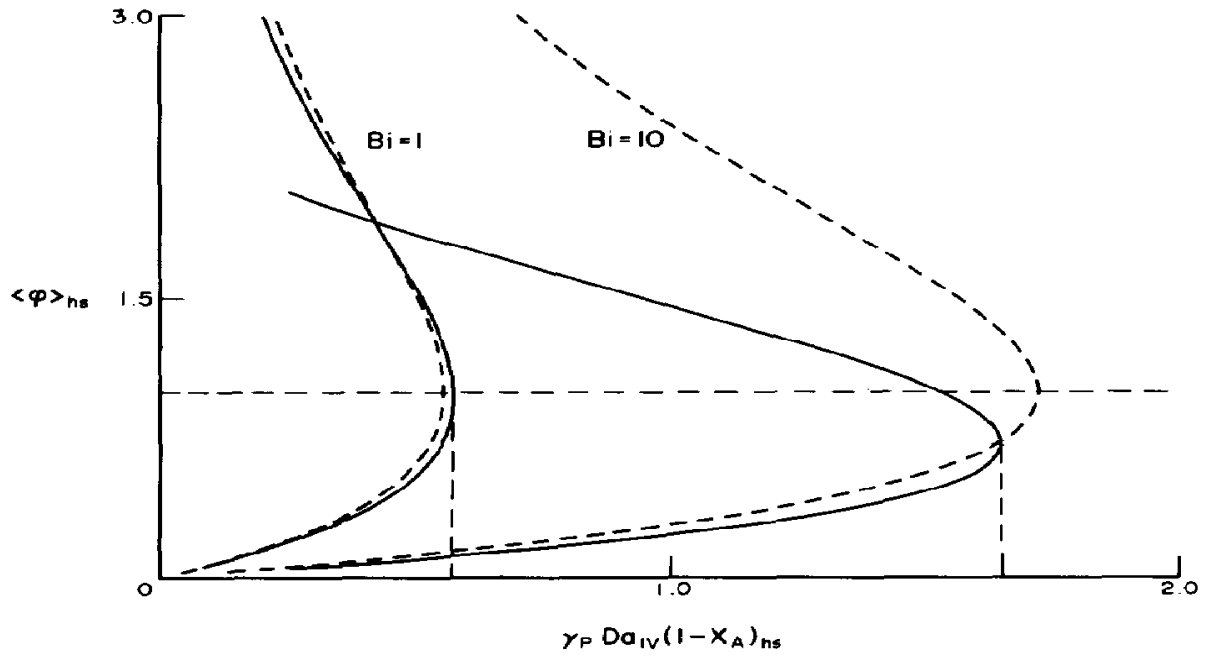

Fig. 6. Loci of maxima curves for the same parameters as in Fig. 4, except that here 3.06 is used as the lumping factor.

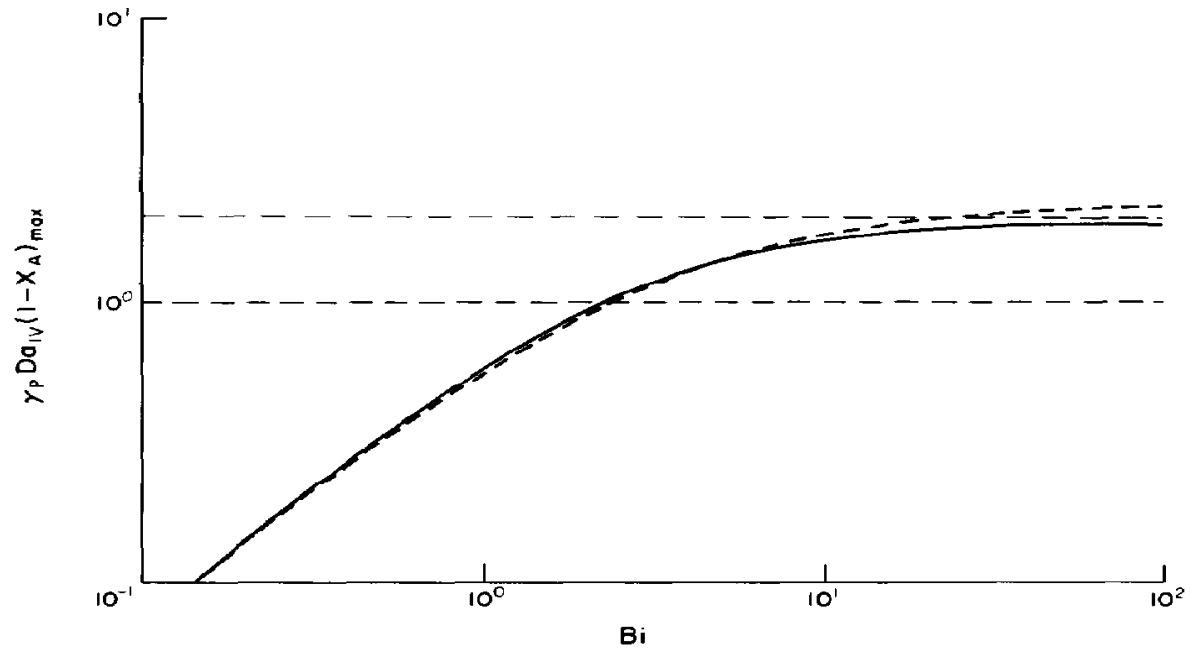

Fig. 7. $\left[\gamma_{P} D a_{I V}\left(1-\left\langle X_{A}\right\rangle\right)_{h s}\right]_{\max }$ as a function of $B i$ and for a lumping factor of 3.06. Further see data of Fig. 5 .

$B i$ values the region is slightly underestimated whereas for high $B i$ values the region is somewhat overestimated.

From the discussions given above we may conclude that the ODM can be used to predict the region of low parametric sensitivity as long as eq. (5) is used to lump the heat transfer parameters.

We will now quantify the differences in hot spot temperature as predicted by the ODM and the RTPM. To this end we introduce a parameter $\Delta_{\theta}$ that accounts for the difference. It is defined as

$$
\begin{aligned}
\Delta_{\theta} & =\left|\frac{\langle T\rangle_{\mathrm{RTPM}}-\langle T\rangle_{\mathrm{ODM}}}{\langle T\rangle_{\mathrm{RTPM}}}\right| \\
& =\left|\frac{\langle\varphi\rangle_{\mathrm{RTPM}}-\langle\varphi\rangle_{\mathrm{ODM}}}{\langle\varphi\rangle_{\mathrm{RTPM}}+\gamma_{P}}\right| .
\end{aligned}
$$

In Fig. 8 the relative error $\Delta_{\theta}$ is plotted vs $\gamma_{P} D a_{\mathrm{IV}}(1$ $\left.-\left\langle X_{A}\right\rangle\right)_{h s}$ for some values of $\gamma_{p}$ and two values of $B i$. Only the lower branch of the locus of maxima curve has been used so that the lines end as soon as the maximum in the locus is reached. We can see that in Fig. 8, where we used eq. (5) for lumping, the error remains below $2 \%$. Using eq. (6) for lumping and plotting values of $\Delta_{\theta}$ lead to Fig. 9: we see that the error is about the same for both lumping relations. Based on these results we may conclude that in the lower branch the ODM gives proper values for the mean temperature, as long as the constant $A$ in eq. (13) has real roots. This agrees with the conclusion of Hlavacek (1970) that the ODM can be used as long as the TDM exhibits no parametric sensitivity. Notice that, although the error made in the hot spot temperature-by using either 3.06 or 4.0 as a lumping 


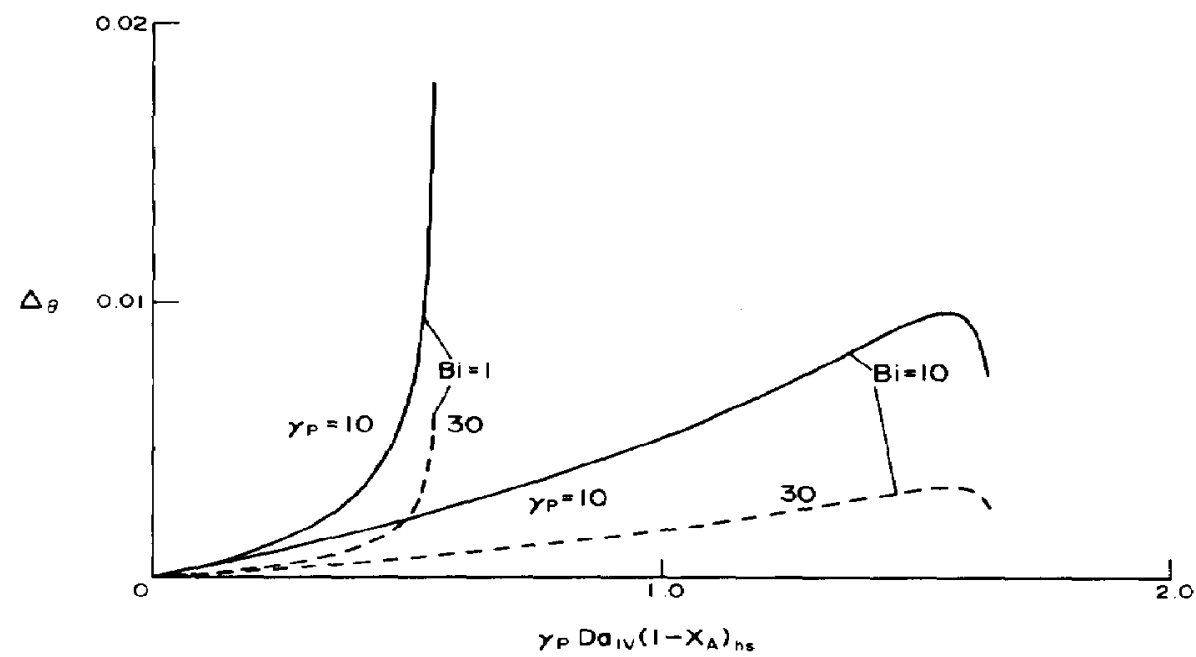

Fig. 8. Relative difference $\Delta_{\theta}$ between the mean hot-spot temperatures as calculated by the ODM and the RTPM for $B i=1$ and 10 and for $\gamma_{F}=10(-\ldots)$ and $30(-\ldots,-$ ). The lumping factor is 4.0. The curves end as soon as $\left[\gamma_{P} D a_{I V}\left(1-\left\langle X_{A}\right\rangle\right)_{h s}\right]_{\max }$ is reached.

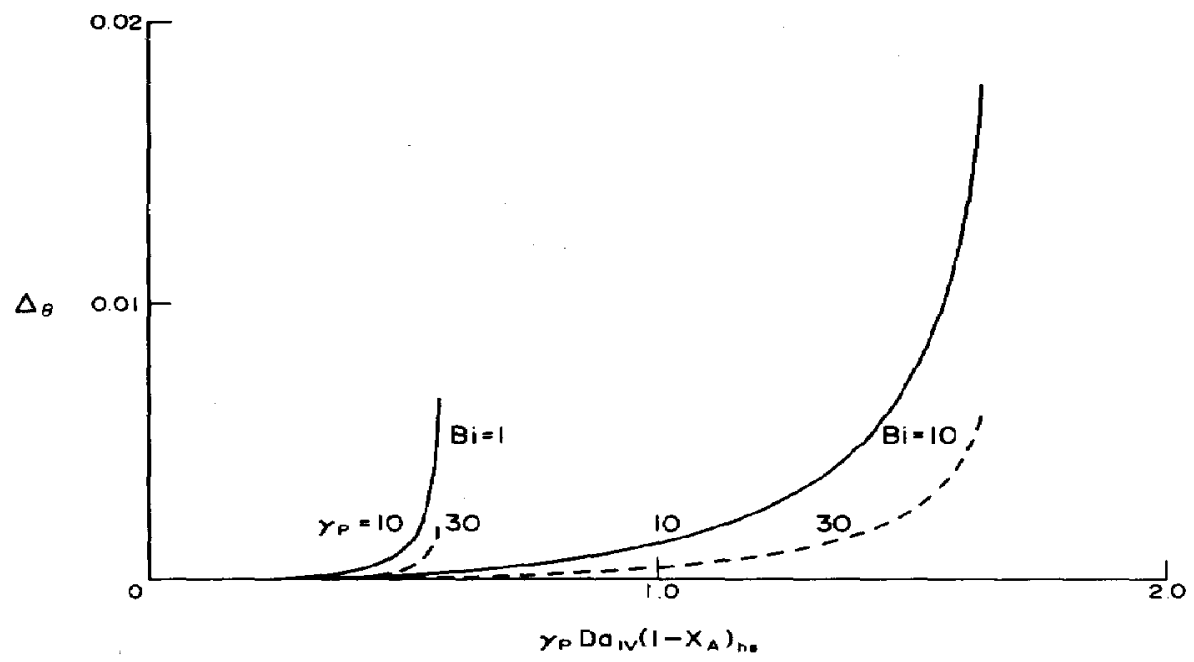

Fig. 9. As Fig. 8 but for the lumping factor 3.06.

factor-is about the same, the factor $\mathbf{3 . 0 6}$ still should be used because it leads to a proper prediction of the region of low parametric sensitivity.

The maximum radial temperature vs the mean temperature

Although the ODM can be used to calculate proper values for the mean temperature in the hot spot, according to the conclusions above, large differences can be found between the mean and the maximum temperature in the hot spot. These differences may lead to an unexpected decrease in selectivity, sintering of the catalyst or even a local runaway. For a correct application of the ODM it is not enough that the ODM predicts proper values for the mean tem- perature. The difference between mean and maximum temperature should also be kept within certain limits.

The maximum radial temperature is achieved in the axis of the reactor tube, so that the maximum temperature in the entire reactor is reached in the center of the hot-spot location. Therefore we will present a method correlating the mean radial temperature in the hot spot to the maximum temperature in the hot spot. This method enables the design engineer to use the ODM and to check whether the profile in the hot spot is flat or rather pronounced.

Using the relation for the mean radial temperature profile [eq. (14)] the mean radial temperature can be calculated when both the integration constant $A$ and the model parameter $\gamma_{P} D a_{I V}\left(1-\left\langle X_{A}\right\rangle\right)_{h a}$ are 
known. For the maximum radial temperature in the hot spot from eq. (12) with $r *=0$ we find that

$$
A=\exp \left(-\varphi_{\max } / 2\right) \text {. }
$$

So for a given value of $\varphi_{\max }$ the value of $A$ may be substituted in the relation for the mean radial temperature. Also the boundary condition should be adhered to, so that the following relation should hold as well:

$$
\begin{aligned}
& \frac{2}{\frac{8 A^{2}}{\gamma_{P} D a_{\mathrm{IV}}\left(1-\left\langle X_{A}\right\rangle\right)_{h s}}+1} \\
& +B i \ln \left[A+\frac{\gamma_{P} D a_{\mathrm{IV}}\left(1-\left\langle X_{A}\right\rangle\right)_{h s}}{8 A}\right]=0
\end{aligned}
$$

The value of $\gamma_{P} D a_{\mathrm{iv}}\left(1-\left\langle X_{A}\right\rangle\right)_{h s}$ is calculated using this equation and a given value of $B i$. Now the mean temperature can be obtained by means of eq. (14) for the given set of values of $\varphi_{\max }$ and $B i$. In Fig. 10 values for the maximum temperature $\varphi_{\max }$ are plotted vs the mean radial temperature $\langle\varphi\rangle$ for some values of $B i$. Of course, the line given by $\varphi_{\max }=\langle\varphi\rangle_{h s}$ corresponds to a completely flat temperature profile found for the value of $B i=0$. For increasing values of $B i$ the temperature profile becomes more pronounced and differences of $T_{\max }-T_{c}$ to $\langle T\rangle-T_{c}$ up to $50 \%$ are possible in the industrial range of $B i$ values.

From Fig. 10 we may conclude that the difference between the mean and maximum radial temperature in the hot spot is much more important than the deviation of the mean temperatures as calculated by the ODM and the RTPM. For the design engineer Fig. 10 is important since it allows him to estimate the mean temperature using the ODM and then the maximum temperature. For a too high maximum temperature he can alter his design and recalculate the reactor. So the two most important temperature values, the mean and the maximum temperature in the hot spot, are found without using the TDM. Using the ODM and Fig. 10 the design engineer can answer all questions about the maximum temperature achieved somewhere in the reactor under stable operating conditions.

\section{Radial differences in reaction rate}

For the design engineer not only the temperature but also the reaction rate is important. If there is a large difference in reaction rate, the selectivity will show large differences as well. Therefore we should also investigate differences in reaction rate profiles. Since there are no radial concentration gradients in the region of low parametric sensitivity the radial reaction rate profile is only affected by the temperature. We will use the same approach for studying the reaction rate profiles as we used for the temperature profiles. First we study the difference between the mean radial reaction rate as obtained from the RTPM and the reaction rate at mean temperature as calculated from the ODM. Next we discuss the reaction rate at the mean temperature in relation to the maximum reaction rate achieved on the reactor axis.

The locus of the mean reaction rate

In the hot spot the reaction rate calculated from the $O D M$ is a reaction rate at the mean temperature and concentration, so we get the following dimensionless form for the reaction rate obtained from the ODM:

$$
\left\langle R_{A}^{*}\right\rangle /\left(1-\left\langle X_{A}\right\rangle\right)_{h s}=\exp \left(\frac{\gamma_{P}\langle\theta\rangle}{1+\langle\theta\rangle}\right)
$$

In Fig. 11 this relation is plotted as a function of the parameter $\gamma_{P} D a_{\mathrm{JV}}\left(1-\left\langle X_{A}\right\rangle\right)_{h s}$. Putting the value $\gamma_{P}=\infty$ is equivalent to using the Frank-Kamenetski approximation, so $\left\langle R_{A}^{*}\right\rangle /\left(1-\left\langle X_{A}\right\rangle\right)_{h s}=\exp \langle\varphi\rangle$. In the ODM the reaction rate is evaluated at the mean temperature, but evidently this is not correct, so we

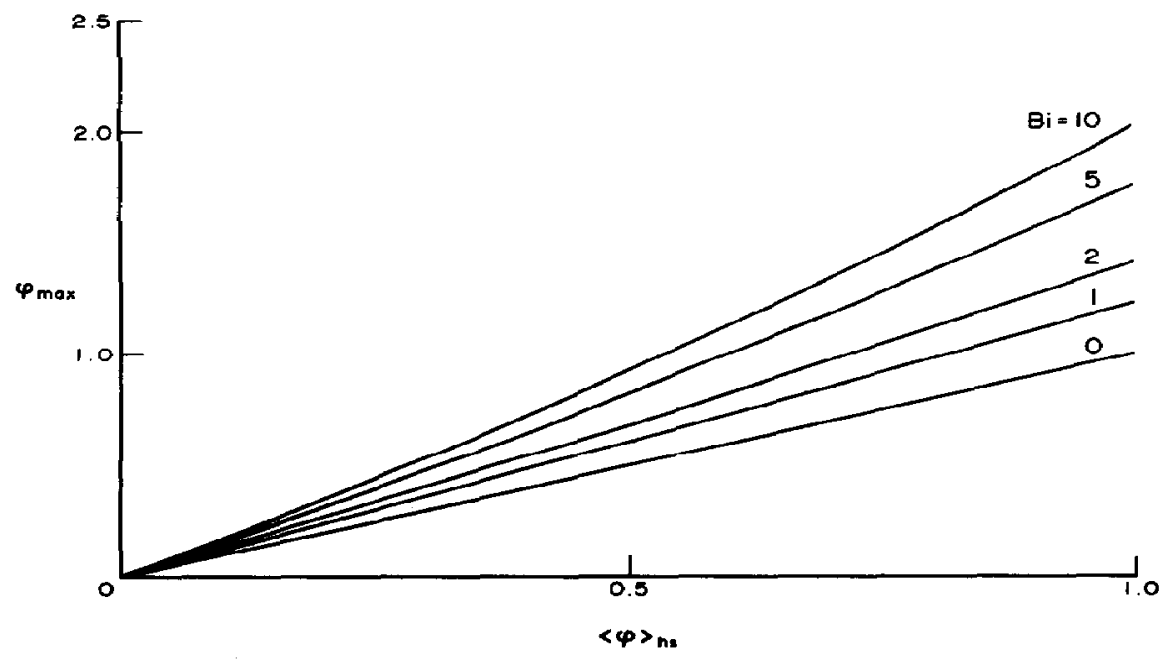

Fig. 10. Maximum temperature vs mean temperature in the hot spot for $B i=0,1,2,5$ and 10 . 


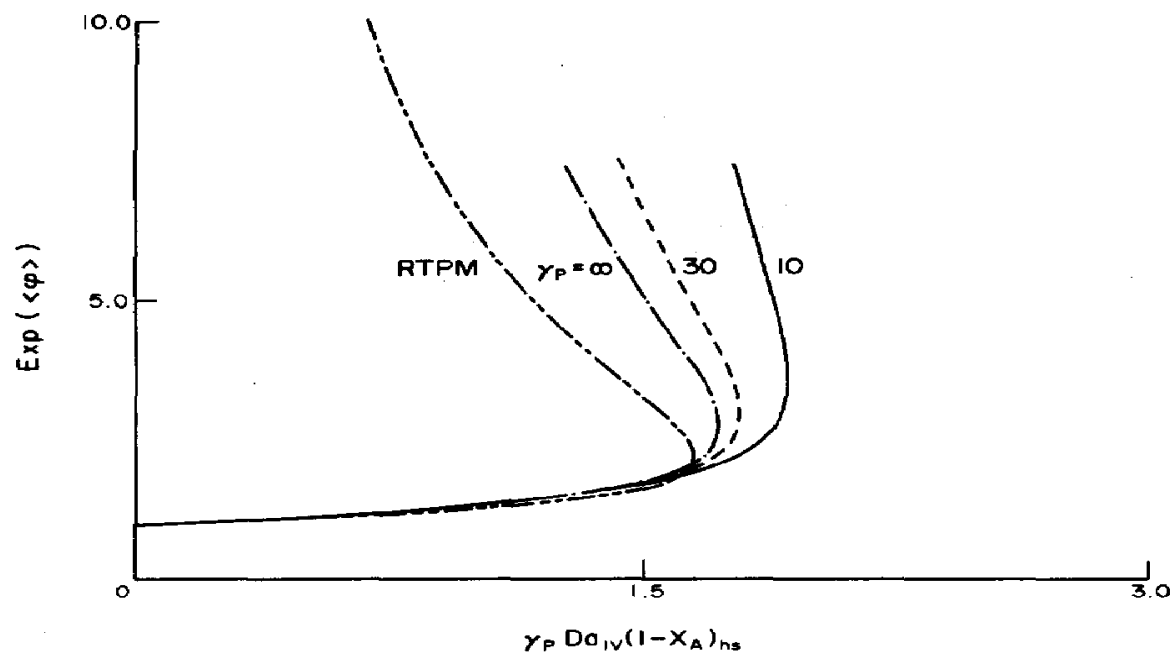

Fig. 11. Mean reaction rate in the hot spot defined by the ODM and represented by $\left\langle R^{*}\right\rangle /\left(1-\left\langle X_{A}\right\rangle\right)_{h s}$ $=\exp (\langle\varphi\rangle)$, as a function of $\gamma_{P} D a_{1 v}\left(1-\left\langle X_{A}\right\rangle\right)_{h s}$ for $\gamma_{P}=10(-)$ and $30(---)$ at Bi $=10$. Also the curves for the ODM (_- - _ - with $\gamma_{P}=\infty$ and the RTPM (- $-\ldots$ ) are given.

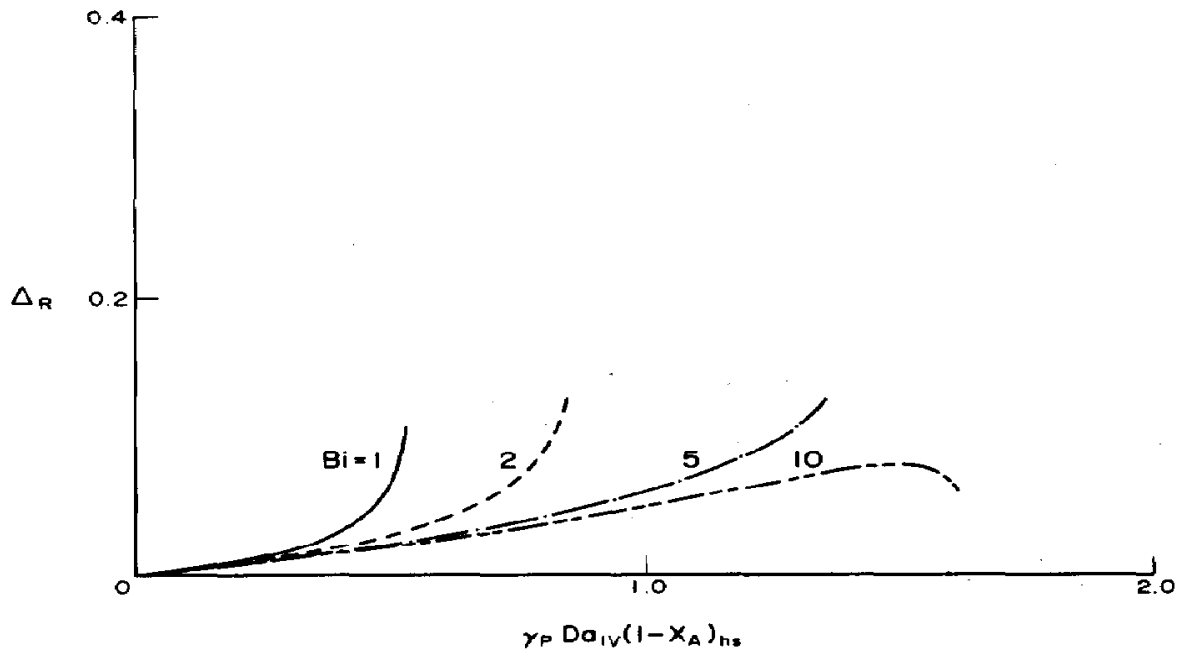

Fig. 12. Difference $\Delta_{R}$ between the reaction rate as calculated by the ODM and the mean reaction rate as calculated by the RTPM for several values of Bi. Here the Frank-Kamenetski approximation is used to obtain the reaction rates at mean temperatures for the ODM.

also calculated the mean reaction rate from the RTPM according to

$$
\begin{aligned}
2 \int_{0}^{1} r^{*} \exp (\varphi) \mathrm{d} r^{*}=\frac{-8 A}{\gamma_{P} D a_{\mathrm{IV}}\left(1-\left\langle X_{A}\right\rangle\right)_{h s}} \\
\times\left[\frac{1}{A+\gamma_{P} D a_{\mathrm{IV}}\left(1-\left\langle X_{A}\right\rangle\right)_{h s} 8 A}-\frac{1}{A}\right]
\end{aligned}
$$

which is equal to $\left\langle R^{*}\right\rangle /\left(1-\left\langle X_{A}\right\rangle\right)_{h s}=\langle\exp (\varphi)\rangle$. Also the locus given by this equation is plotted in Fig. 11. It is clear that the discrepancy between the ODM and the RTPM can be large since the difference in temperature is magnified by the exponential factor. Similar to the relative error in temperatures between the ODM and the RTPM we define a factor $\Delta_{R}$ which makes it possible to compare the differences in reaction rate. It is defined as

$$
\Delta_{R}=\left|\frac{2 \int_{0}^{1} r^{*} \exp \left(\varphi_{\mathrm{RTPM}}\right) \mathrm{d} r^{*}-\exp \left(\left\langle\varphi_{\mathrm{ODM}}\right\rangle\right)}{2 \int_{0}^{1} r^{*} \exp \left(\varphi_{\mathrm{RTPM}}\right) \mathrm{d} r^{*}}\right|
$$

In Fig. 12 we plotted the factor $\Delta_{R}$ vs $\gamma_{P} D a_{I V}$ $\left(1-\left\langle X_{A}\right\rangle\right)_{h s}$ using 3.06 as the lumping factor for the heat transfer parameters. From Fig. 12 it can be concluded that the error in the mean reaction rate can be as large as $15 \%$, which is much higher then the error made in the temperature. Based on this conclusion it 


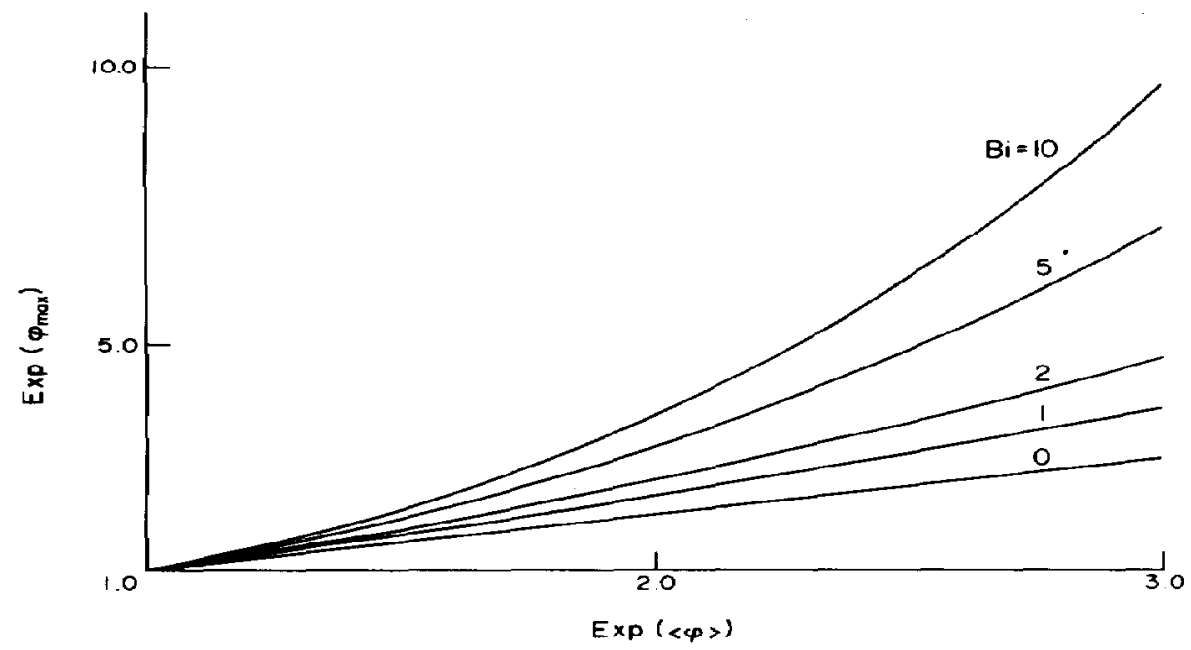

Fig. 13. Maximum reaction rate as a function of the reaction rate at the mean temperature both determined with the RTPM for $B i=0,1,2,5$ and 10 .

follows that when the ODM is used for multiple reactions the error in the selectivity can be significant. However, kinetic data are seldom known with an accuracy better than $15 \%$. Therefore, we still advise using the ODM for preliminary design procedures.

The maximum reaction rate $v$ s the reaction rate at mean temperature

Like for the mean temperature and the maximum temperature in the axis at the hot spot we can also relate the maximum reaction rate to the reaction rate at mean temperature as obtained with the ODM. Using this relation one obtains an indication for the differential selectivity profile in the hot spot. In case of a large difference between the mean and the maximum rate the selectivity achieved can be much lower then estimated from the results of the ODM. In Fig. 13 we plotted the maximum reaction rate $\varphi_{\max }$ vs the reaction rate at mean temperature for various values of $B i$. The differences can be considerable: for high $B i$ values-so for pronounced temperature profilesthey can amount to $100 \%$. In view of these results we may expect serious effects of the actual temperature profile on the local selectivity. An extensive discussion of the effect of the temperature profile on the local and mean selectivity is given by Westerink (1988).

\section{CONCLuSIONS}

In this study we discussed the two most frequently used models for the cooled tubular reactor, the ODM and the TDM, and answered the question when the $O D M$ can be used for preliminary design studies. We restricted ourselves to study effects in the hot spot only, since here the effects are most extreme. From numerical evaluations of the TDM it was found that no significant concentration gradients exist for reactors operating under industrial conditions; also it was found that the hot spot is located at one axial position. Based on these results we developed the
RTPM model that describes the radial temperature profile in the hot spot analytically for single reactions. From numerical evaluations again the RTPM and the TDM proved to be equivalent in the region of low parametric sensitivity. When the factor $\gamma_{P} D a_{I V}(1$ $\left.-\left\langle X_{A}\right\rangle\right)_{h s}$ was calculated from the conversion obtained from the ODM the results were even better. Based on this property we may state that the TDM can be replaced by the ODM together with the RTPM when effects in the hot spot are subject of studies. For these studies the Frank-Kamenetski approximation should hold true and the solution of the parameters should fall in the region of low parametric sensitivity.

In our discussion of the applicability of the ODM we distinguished two criteria for comparison:

(1) the temperature. The mean temperature in the hot spot was compared with the temperature obtained from the ODM. It was found that the ODM leads to reliable values for the mean temperature as long as the solution of the boundary condition for the RTPM [eq. (13)] has real roots. We also compared the difference in the mean and the maximum temperature and found that differences up to $50 \%$ of $T-T_{c}$ are possible. Moreover, we showed that the lumping relation presented by Crider and Foss (1965) can be used to predict the region of low parametric sensitivity. However, their factor of 3.06 in the lumping relation is not that strict and can be put equal to 3 .

(2) reaction rates. The ODM and the RTPM were compared with respect to reaction rates and it was found that even for reactors operating in the region of low parametric sensitivity there can be large differences in the mean reaction rate as obtained from the RTPM and the reaction rate at mean temperature as calculated 
from the ODM. From this it follows that radial differences in reaction rate can be significant. However, one should realize that kinetic data are usually known with an accuracy less than $15 \%$.

Based on the discussion above we advise to use the ODM for preliminary design procedures and check the results with an extensive study using the TDM.

\section{NOTATION}

$A$ integration constant in eq. (13)

$B i$ Biot number for heat transfer through the wall $\left(\alpha_{w} R_{t} / \lambda_{\text {erf }}\right)$

$B o_{h} \quad$ Bodenstein number for heat $\left[v_{o}\left(\rho_{g} C_{p}\right)_{q} D_{p} /\right.$ $\lambda_{\text {eff }]}$

$B o_{m} \quad$ Bodenstein number for mass $\left(v_{o} D_{p} / D_{\text {eff }}\right)$

$C_{i} \quad$ concentration of species $i, \mathrm{~mol} / \mathrm{m}^{3}$

$C_{p} \quad$ specific heat of the reaction mixture, $\mathrm{J} /(\mathbf{k g ~ K})$

$D_{\text {eff }} \quad$ effective radial diffusion coefficient for mass, $\mathrm{m}^{2} / \mathrm{s}$

$D a_{I} \quad$ first Damköhler number, used for residence times $\left(k_{\mathrm{c}} L / v_{o}\right)$

$D a_{\text {Iv }}$ fourth Damköhler number $\left[k_{c}\left(-\Delta H_{P}\right)\right.$ $\left.\left\langle C_{A_{0}}\right\rangle R_{t}^{2} /\left(\lambda_{\text {eff }} T_{c}\right)\right]$

$D_{p} \quad$ diameter of the catalyst particle, $\mathbf{m}$

$D R$

$D_{\mathbf{t}}$

$\boldsymbol{E}_{\boldsymbol{i}}$

$\Delta H_{i}$ reaction enthalpy for the reaction producing ratio of the diameter of the catalyst particle and the tube $\left(D_{p} / D_{t}\right)$

diameter of the tube, $m$ product $i, J / \mathrm{mol}$

$k_{i} \quad$ reaction rate constant for the reaction producing $i, s^{-1}$

$L \quad$ length of the reactor tube, $m$

$L R$ length to diameter ratio of the reactor tube $\left(L / D_{t}\right)$

ODM one-dimensional model of the cooled tubular reactor

$r \quad$ radial coordinate, $m$

$r^{*}$ dimensionless radial coordinate $\left(\boldsymbol{r} / \boldsymbol{R}_{\mathrm{t}}\right)$

$\boldsymbol{R}$ gas constant, $\mathbf{J} /(\mathbf{m o l} \mathbf{K})$

$R_{i}^{*}$ dimensionless reaction rate $\left[R_{t} /\left(k_{c} C_{A_{0}}\right)\right]$

$\boldsymbol{R}_{i} \quad$ reaction rate of the reaction producing product $i, \mathrm{~mol} /\left(\mathrm{m}^{3} \mathrm{~s}\right)$

$\boldsymbol{R}_{\text {, }} \quad$ radius of the reactor tube, $m$

RTPM radial temperature profile model in the hot spot for one single reaction

$T$ temperature, $\mathbf{K}$

$\langle T\rangle$ mean temperature, $\mathrm{K}$

TDM two-dimensional model of the cooled tubular reactor

$U$ overall heat transfer coefficient, $W /\left(m^{2} \mathrm{~K}\right)$

$U$ * dimensionless heat transfer coefficient $\left[4 U /\left(\rho_{g} k_{c} C_{p g} D_{t}\right)\right]$

$v_{o} \quad$ superficial gas velocity based on the packed cross section of the bed, $\mathrm{m} / \mathrm{s}$

$X_{i} \quad$ conversion of species $i\left[\left(C_{i o}-C_{i}\right) / C_{i o}\right]$ dimensionless axial coordinate $(Z / L)$

axial coordinate, $m$

Greek letters

$\alpha_{w}$ heat transfer coefficient at the wall, $\mathbf{W} /\left(\mathbf{m}^{2} \mathbf{K}\right)$

$\gamma_{t}$ dimensionless temperature of activation $\left[\left(E_{i} / R T_{c}\right)\right]$

$\Delta$ difference between values obtained from the ODM and the RTPM

$\varepsilon$ porosity of the packed tube

$\eta_{g} \quad$ viscosity of the gas mixture, $\mathrm{N} \mathrm{s} / \mathrm{m}^{2}$

$\theta$ dimensionless temperature $\left[\left(T-T_{c}\right) / T_{c}\right]$

$\langle\theta\rangle$ mean temperature over the radius $[(\langle T\rangle$ $\left.\left.-T_{c}\right) / T_{c}\right]$

$\Delta \theta_{\mathrm{ad}}$ dimensionless adiabatic temperature rise $\left[-\Delta H_{p} C_{A o} /\left(\rho_{q} C_{p} T_{c}\right)\right]$

$\lambda_{\text {eff }} \quad$ effective heat conductivity in radial direction, $\mathbf{W} /(\mathbf{m ~ K})$

density of the gas mixture, $\mathrm{kg} / \mathrm{m}^{3}$

$\rho$

$\varphi$ modified dimensionless temperature $\left(\gamma_{P} \theta\right)$

$\langle\varphi\rangle$ modified mean temperature $\left(\gamma_{P}\langle\theta\rangle\right)$

\section{Subscripts}

A reactant

c at coolant temperature

$g$ of the gas mixture

$h$ for heat

$m$ for mass

max maximum

o at inlet conditions

$p \quad$ of the particle

$P$ desired reaction product

$R$ for reaction ratcs

\section{REFERENCES}

Beek, J., 1962, Design of packed catalytic reactors, in Advances in Chemical Engineering (Edited by Th. Drew, J. W. Hoopes and Th. Vermeulen), Vol. 3, pp. 203-271. Academic Press, New York.

Barkelew, C. H., 1955, Stability of chemical reactors. Chem. Engng Prog. Symp. Ser. 55, 37-46.

Chambré, P. L. and Grossman, L. M., 1955, On limiting temperatures in chemical reactors. Appl. Sci. Res. A5, 245-254.

Crider, J. E. and Foss, A. S., 1965, Effective wall heat transfer coefficients and thermal resistances in mathematical models of packed beds. A.I.Ch.E. J. 11, 1012-1019.

Dixon, A. G. and Cresswell, D. L., 1979, Theoretical prediction of effective heat transfer parameters in packed beds. A.I.Ch.E. J. 25, 663-676.

Fahien, R. W. and Smith, J. M., 1955, Mass transfer in packed beds. A.I.Ch.E. J. 1, 28-37.

Froment, G. F., 1967, Fixed bed catalytic reactors: current design status. Ind. Engng Chem. 59, 18-27.

Froment, G. F., 1974, Fixed bed catalytic reactors: technological and fundamental design aspects. Chemie-Ingr-Tech. 46, 374-386.

Hennecke, F. W. and Schlünder, E. U., 1973, Wärmeübergang in beheizten oder gekühlten Rohren mit Schüttungen aus Kugeln, Zylindern und Raschig Ringen. Chemie-IngrTech. 45, 277-284.

Hlavacek, V., 1970, Aspects in design of packed catalytic reactors. Ind. Engng Chem. 62(7), 8-26.

Morbidelli, M. and Varma, A., 1988, A generalized criterion 
for parametric sensitivity: application to thermal explosion theory. Chem. Engng Sci. 43, 91-102.

Morbidelli, M. and Varma, A., 1989, A generalized criterion for parametric sensitivity: application to a pseudo-homogeneous tubular reactor with consecutive or parallel reactions. Chem. Engng Sci. 44, 1675-1696.

Mears, D. E., 1971, Diagnostic criteria for heat transport limitations in fixed bed reactors. $J$. Catal. 20, 127-131.

Westerink, E. J., 1988, Heat effects in chemical reactors: design and stability. PhD thesis, Twente University, Enschede.

Westerink, E. J. and Westerterp, K. R., 1988, Safe design of cooled tubular reactors for exothermic multiple reactions: multiple reaction networks. Chem. Engng Sci. 43, 1051-1069.

Westerterp, K. R. and Overtoom, R. R. M., 1985, Safe design of cooled tubular reactors for exothermic multiple reactions; consecutive reactions. Chem. Engng Sci. 40, 155-165.

Westerterp, K. R. and Ptasinsky, K. J., 1984a, Safe design of cooled tubular reactors for exothermic multiple reactions; parallel reactions. Development of criteria. Chem. Engng Sci. 39, 235-245.

Westerterp, K. R. and Ptasinsky, K. J., 1984b, Safe design of cooled tubular reactors for exothermic multiple reactions; parallel reactions. The design and operation of an ethylene oxide reactor. Chem. Engng Sci. 39, 245-252.

Westerterp, K. R., van Swaaij, W. P. M. and Beenackers, A. A. C. M, 1984, Chemical Reactor Design and Operation, pp. 305-307, John Wiley, New York.

Zehner, P. and Schlünder, E. U., 1973, Die effektive Wärmeleitfähigkeit durchströmter Kugelschüttungen bei mäBigen und hohen Temperaturen. Chemie-Ingr-Tech. 45, 272-276.

\section{APPENDIX 1: SOLUTION OF DIFFERENTIAL EQ. (11)}

For the radial temperature profile in the hot spot of the reactor the following nonlinear differential equation has to be solved:

$$
-\frac{1}{x} \frac{\mathrm{d}}{\mathrm{d} x}\left(x \frac{\mathrm{d} y}{\mathrm{~d} x}\right)=S \exp (y)
$$

where $S=\gamma_{P} D a_{\mathrm{IV}}\left(1-\left\langle X_{A}\right\rangle\right)$ and the boundary conditions are

$$
\left.\frac{\mathrm{d} y}{\mathrm{~d} x}\right|_{x=1}=\left.B i y\right|_{x=1} \text { and }\left.\frac{\mathrm{d} y}{\mathrm{~d} x}\right|_{x=0}=0 .
$$

Equation (A1) can be rewritten as $x y^{\prime \prime}+y^{\prime}+x S \exp (y)=0$. Introducing the two variables $u(t)=x y^{\prime}(x)$ and $t$ $=x^{2} \exp (y)$ leads to the following relations:

$$
y^{\prime}(x)=u(t) / x \quad \text { and } \quad y^{\prime \prime}(x)=\left[\frac{u(t)}{x} \frac{\mathrm{d} t}{\mathrm{~d} x}-\frac{u(t)}{x^{2}}\right]
$$

Moreover $\quad \frac{\mathrm{d} t}{\mathrm{~d} x}=[u(t)+2] x \exp (y)$.

Substitution of the new variables into the difierential equation yields

$$
[u(t)+2] u^{\prime}(t)+S=0 .
$$

The solution is

$$
u^{2}(t)+4 u(t)=-2 S t-4 A .
$$

Substitution of $x$ and $y$ gives

$$
x^{2}\left[y^{\prime}(x)\right]^{2}+4 x y^{\prime}(x)=-2 S x^{2} \exp (y)-4 A .
$$

We now have two equations [(A1) and (A2)] and two unknown variables $(y$ and $A$ ). Eliminating the exponential term in both equations, we obtain

$$
-2 x^{2} y^{\prime \prime}+x^{2}\left(y^{\prime}\right)^{2}+2 x y^{\prime}+4 A=0 .
$$

Once more we introduce a new variable, $y^{\prime}=-2 v^{\prime} / v$, so that $y^{\prime \prime}=-2 v^{\prime \prime} / v+2\left(v^{\prime}\right)^{2} / v^{2}$. Substitution into eq. (A3) leads to $x^{2} v^{\prime \prime}-x v^{\prime}+A v=0$. This is a differential equation of the Euler type and its solution is

$$
v(x)=A_{1} x^{1-\sqrt{1-A}}+A_{2} x^{1}+\sqrt{1-A} \text {. }
$$

From the definition of $v$ it follows that $y=-2 \ln |v|+A_{3}$, so the final solution in terms of the variables $x$ and $y$ becomes

$$
y(x)=-2 \ln \left(A_{1} x^{1-\sqrt{1-A}}+A_{2} x^{1+\sqrt{1-A}}\right) .
$$

The integration constant $A_{3}$ is absorbed in $A_{1}$ and $A_{2}$. We introduce $\alpha=\sqrt{1-A}$. We now have the original differential eq. (A1) and its solution [eq. (A4)], in which the original parameters $x$ and $y$ are present as well as the unknown integration constants $A_{1}, A_{2}$ and $\alpha$. To solve for the value of $\alpha$ we substitute eq. (A4) into the differential eq. (A1). This leads to the following relation between $\alpha, A_{1}$ and $A_{2}$ :

$$
\alpha= \pm \sqrt{S / 8 A_{1} A_{2}} \text {. }
$$

Now in terms of the real integration constants the solution becomes

$$
\begin{aligned}
y(x)= & -2 \ln \left(A_{1} x^{1}-\sqrt{S / 8 A_{1} A_{2}}\right. \\
& \left.+A_{2} x^{1}+\sqrt{S / 8 A_{1} A_{2}}\right) .
\end{aligned}
$$

From the second boundary condition, which demands that the solution of the differential equation is even, only even powers of the polynomial are possible. This means that $\alpha$ can only be equal to 1 , so the solution simplifies to

$$
y(x)=-2 \ln \left(A_{1}+A_{2} x^{2}\right) \text { and } A_{2}=S /\left(8 A_{1}\right)
$$

so that

$$
y(x)=-2 \ln \left[A_{1}+S /\left(8 A_{1}\right) x^{2}\right] .
$$

In terms of the dimensionless temperature $\theta$ and the dimensionless radius $r *$, the solution becomes

$$
\theta=-\frac{2}{\gamma_{P}} \ln \left[A_{1}+\frac{S}{8 A_{1}}\left(r^{*}\right)^{2}\right] .
$$

Using the first boundary condition we obtain the following implicit equation for the determination of $A_{1}$ :

$$
\frac{2}{\frac{8 A_{1}^{2}}{S}+1}+B i \ln \left(A_{1}+\frac{S}{8 A_{1}}\right)=0 .
$$

Equations (A5) and (A6) together describe the radial temperature profile in the hot spot of a cooled tubular reactor. 